\title{
Fulminant hepatic failure following ingestion of wild mushrooms
}

\author{
Corey M. Stein MD, Peter E. Wu MD, James A. Scott PhD, Adina S. Weinerman MD
}

Competing interests: None declared.

This article has been peer reviewed.

The authors have obtained patient consent.

Correspondence to: Adina Weinerman, adina. weinerman@sunnybrook.ca

CMAJ 2015. DOI:10.1503 /cmaj.150080
A previously healthy 52-year-old immigrant woman of Asian descent presented to the emergency department in early August with acute-onset abdominal pain, nausea, vomiting and watery diarrhea. About 12 hours earlier, she had eaten wild mushrooms that she had harvested from a local park with her husband, who had previous foraging experience in his native land. She brought a sample of the mushrooms with her to the emergency department. Nobody else had eaten the mushrooms. The patient had no other important medical history and was not taking prescription medications, over-the-counter medications or alternative supplements.

On arrival at the emergency department, the patient was hemodynamically stable, with a blood pressure of 124/79 $\mathrm{mm} \mathrm{Hg}$ and a heart rate of 81 beats/min. She appeared dehydrated. She was awake and fully oriented but was in obvious discomfort. Her epigastrium was tender to palpation, with no peritoneal signs. There was no jaundice or asterixis. Cardiac and respiratory examinations yielded normal findings. Initial blood work revealed slightly elevated aminotransferase levels. The international normalized ratio and levels of bilirubin and albumin were within normal limits.

The patient was rehydrated with $0.9 \%$ sodium chloride given intravenously. She was also given morphine and ondansetron intravenously for relief of symptoms. Activated charcoal was given at a dose of $50 \mathrm{~g}$ orally every four hours to enhance toxin elimination, given the suspicion of possible mushroom toxicity. Because of its hepatoprotective effects, empiric treatment with $\mathrm{N}$-acetylcysteine was started by continuous intravenous infusion at a loading dose of $150 \mathrm{mg} / \mathrm{kg}$ over 1 hour, followed by $50 \mathrm{mg} / \mathrm{kg}$ over 4 hours and then $100 \mathrm{mg} / \mathrm{kg}$ over 16 hours, for a total dose of $300 \mathrm{mg} / \mathrm{kg}$ over 21 hours. The patient was admitted to the internal medicine service.

The following morning, the mushrooms ingested by the patient were identified as Amanita bisporigera, a known hepatotoxic species. Local public health authorities were contacted to help identify the location of the mushrooms and prevent further public consumption. Meanwhile, the patient's transaminase levels and liver function progressively worsened (Appendix 1, available at www.cmaj.ca/lookup/suppl/doi:10.1503/cmaj $.150080 /$-/DC1). About 36 hours after eating the mushrooms, she was transferred to the intensive care unit, and the on-call liver transplant team was contacted.

The following day, the patient's clinical status continued to worsen. Her level of consciousness began to deteriorate, consistent with hepatic encephalopathy, and profound coagulopathy developed, with an international normalized ratio of more than 13 . The patient was urgently referred to a liver transplant centre for further management.

An expedited pre-transplant workup was completed, which included echocardiography, abdominal ultrasonography, chest radiography, and appropriate testing for HIV, hepatitis, cytomegalovirus and Epstein-Barr virus. The patient subsequently underwent an urgent liver transplant from a deceased donor. There were no complications associated with the procedure. Her postoperative course was unremarkable aside from pneumonia, for which she was given oral antibiotic treatment. Immunosuppressive therapy (prednisone, tacrolimus and mycophenolate) was started, and the patient was discharged home 10 days after transplantation. 


\section{Discussion}

More than 20000 species of mushrooms are currently recognized, and estimates of up to 1 million undiscovered species have been proposed. ${ }^{1}$ Despite this sizeable biodiversity, only a few hundred named species have been reported as poisonous to humans. ${ }^{2}$ Among the most poisonous, several members of the genus Amanita are responsible for the majority of deaths from mushroom toxicity: these include A. phalloides (accounting for more than $90 \%$ of all deaths), A. verna and $A$. virosa. ${ }^{3}$ The species ingested by our patient was $A$. bisporigera (Figure 1). Like most other Amanita species that exist in temperate latitudes, A. bisporigera is a symbiotic associate of tree roots, in this case oak. Unlike the morphologically similar, though smaller, fall-fruiting species $A$. verna and $A$. virosa, A. bisporigera tends to produce dense flushes of large, strikingly white fruiting bodies during mid-summer in eastern North America. ${ }^{2,3}$

Because of the likely high number of unreported cases, the true incidence of mushroom poisoning is unclear. In the United States, about 6000 exposures to toxic mushrooms are reported annually, most of which are associated with mild symptoms. ${ }^{4}$ In Western Europe, mushroom toxicity is more common, with 50-100 fatal cases reported annually. ${ }^{3}$ When serious toxicity does occur, as in our patient, it is typically related to foraging by adults who misidentify a toxic mushroom with morphology similar to a known edible species.

\section{Pathogenesis}

The two distinct pathogenic toxins isolated from Amanita species are phalloidin and $\alpha$-amanitin (also referred to as an amatoxin). Phalloidin impairs cellular membrane function and induces the initial gastrointestinal symptoms of nausea, vomiting and diarrhea. ${ }^{3}$ Alpha-amanitin is responsible for the severe morbidity and mortality associated with mushroom poisoning and is not denatured by cooking or freezing. ${ }^{3}$ After being absorbed through the intestinal epithelium, $\alpha$-amanitin is transported into hepatocytes, where it inhibits the enzyme RNA polymerase II and thus interrupts the synthesis of structural proteins, which results in massive hepatocellular necrosis. ${ }^{3}$ Although not observed in our patient (Appendix 1), direct nephrotoxic effects by $\alpha$-amanitin at the proximal and distal convoluted tubules can occur, causing acute tubular necrosis. ${ }^{3}$

The lethal dose of $\alpha$-amanitin is $0.1 \mathrm{mg} / \mathrm{kg}$, which can be reached in many cases by ingesting a single mushroom. In addition to Amanita, $\alpha$-amanitin is also found in certain species of the genera Lepiota and Galerina. ${ }^{5}$

\section{Clinical presentation}

The clinical presentation of Amanita toxicity is classically described as triphasic. ${ }^{6}$ The first phase is characterized by a subacute onset (about 6-24 hours after ingestion) of severe gastrointestinal symptoms, including nausea, vomiting, abdominal pain and diarrhea. ${ }^{6,7}$ The second phase lasts 24-48 hours and is a false "recovery" period that could lead to premature discharge of patients. ${ }^{6,7} \mathrm{It}$ is during this phase, however, that $\alpha$-amanitin damages hepatocytes and results in progressive transaminitis. ${ }^{7}$ The third phase occurs after 48 hours and is characterized by hepatic dysfunction. Patients experience a profound coagulopathy and rapidly progressing hepatic encephalopathy. ${ }^{7}$ Renal failure may develop during this phase as a result of hepatorenal syndrome or the direct nephrotoxic effects of $\alpha$-amanitin. ${ }^{7}$

Our patient had a severe presentation without any recovery period, presenting with gastrointestinal symptoms about 12 hours after ingestion. Although her liver enzyme levels were only slightly elevated (well below $100 \mathrm{IU} / \mathrm{L}$ ) at 12 hours after ingestion, her condition progressed rapidly to liver failure.

\section{Management}

Once amatoxin poisoning is diagnosed or suspected, aggressive supportive care, close monitoring of hepatic function and consultation with a local poison control centre for adjunctive therapy are the main principles of management. Hypovolemia, and electrolyte and metabolic derangements as a result of gastrointestinal symptoms are likely. ${ }^{8}$ With the onset of liver failure, coagulopathy and encephalopathy will require management as well as prompt consideration for liver transplantation. ${ }^{6}$

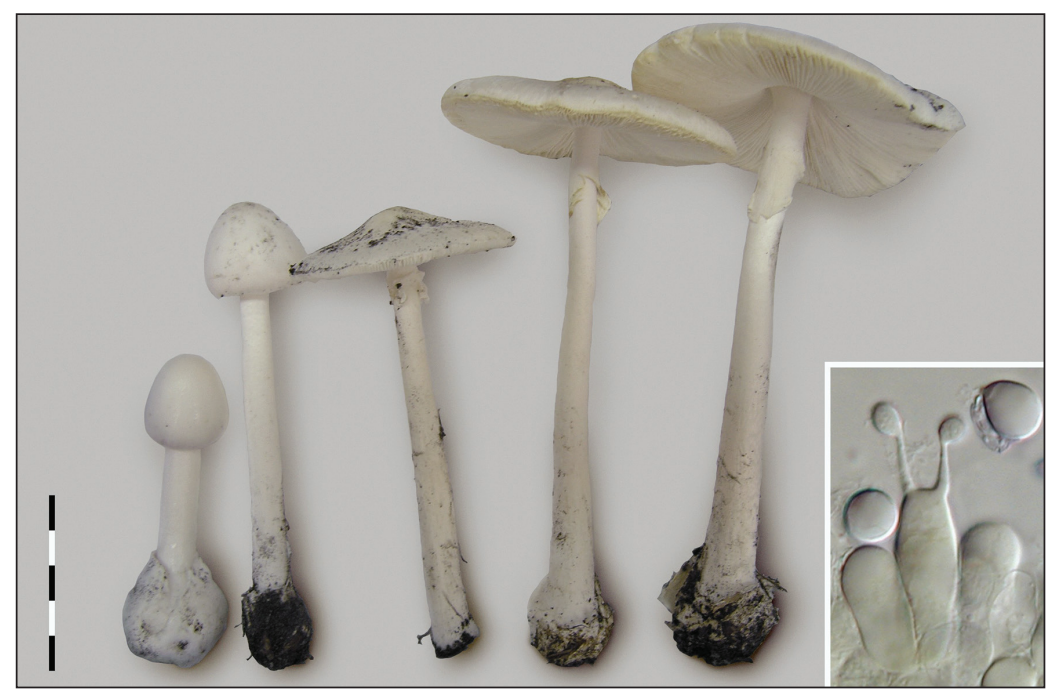

Figure 1: Specimens of Amanita bisporigera at varying stages of maturity collected from the fruiting patch where the patient and her husband had foraged (bar = $5 \mathrm{~cm}$ ). Inset shows the two-spored basidium, which is characteristic of the species (bar $=10 \mu \mathrm{m})$. 
Although the toxin is adsorbed by charcoal, the delayed presentation of patients limits the clinical utility of gastrointestinal decontamination. ${ }^{5}$ To facilitate elimination, multi-dose activated charcoal can be used, because the amatoxin undergoes enterohepatic circulation; however, extracorporeal measures, specifically hemodialysis, have unfortunately proven ineffective in eliminating the toxin. ${ }^{5,6,9}$

There is no specific antidote, and adjunct pharmacologic therapy focuses on the oxidant effects of the toxin as well as its uptake via hepatic transporters. ${ }^{10}$ Many therapies have been studied, with some of the more commonly used options being $N$-acetylcysteine, $\beta$-lactam antibiotics, cimetidine and silibinin. ${ }^{5,6,8-10}$

$\mathrm{N}$-acetylcysteine is used because of its proposed antioxidant and hepatoprotective effects. It is relatively safe, and its potential benefits are generally viewed to outweigh any risks. ${ }^{5,8}$ Use of a high-dose $\beta$-lactam antibiotic, specifically benzylpenicillin (penicillin $\mathrm{G}$ ) or ceftazidime, has been described in case reports, with a purported mechanism of either interference with hepatic uptake or intracellular interruption of the toxin.,5,6 Cimetidine, a general cytochrome P450 (CYP450) inhibitor, has also been used based on an early postulation that amatoxin acts similarly to other CYP-mediated toxins. ${ }^{5,8}$ Lastly, silibinin, a constituent of the milk thistle Silybum marianum, is thought to act by interfering with the hepatic uptake of amatoxin. ${ }^{8}$ Silibinin therapy has been given intravenously in case reports and appears to be well tolerated. ${ }^{5,6,8,9}$ However, it is available in the US only through phase II and III open-label trials as Legalon, and in Canada it is generally not available without a Special Access request form.

None of these or other pharmacologic treatments have been definitively shown to improve outcomes. ${ }^{5,6,8-10}$ Their theoretical benefit is derived from an understanding of the amatoxin pathophysiology. Evidence to date, which is primarily based on case reports and retrospective analyses of poisonings, is inconclusive, and one murine model showed that all of these treatments were ineffective at limiting hepatic injury. ${ }^{10}$ Nevertheless, these treatments are still considered given the potentially fatal consequences of poisoning and the goal of trying to avoid liver transplantation.

Ongoing management and consideration of additional therapies should be made in consultation with the local poison control centre and the patient. Frequent blood work to assess liver parameters should be performed in the first 24-48 hours, because hepatic injury can occur in the absence of any clinical symptoms.

Measures should be undertaken in conjunction with public health authorities to identify the location of the mushrooms and prevent further public consumption.

Distinguishing safe from harmful mushrooms is a challenge even for mycologists. Patients should be counselled that poisonous and edible mushrooms can be very similar in appearance and that wild mushrooms of uncertain identity should not be eaten. This information is especially important for immigrants who might mistake local poisonous mushrooms for familiar edible species from their native land.

\section{References}

1. Kirk PM, Cannon PF, Minter DW, et al. Dictionary of the fungi. 10th ed. Wallingford (UK): CABI Publishing; 2008.

2. Rumack BH, Spoerke DR. Handbook of mushroom poisoning: diagnosis and treatment. Boca Raton (FL): CRC Press; 1994.

3. Broussard CN, Aggarwal A, Lacey SR, et al. Mushroom poisoning - from diarrhea to liver transplantation. Am J Gastroenterol 2001;96:3195-8

4. Bronstein AC, Spyker AD, Cantilena LR, et al. 2008 annual report of the American Association of Poison Control Centers' National Poison Data System (NPDS): 26th annual report. Clin Toxicol (Phila) 2009;47:911-1084.

5. Enjalbert F, Rapior S, Nouguier-Soulé J, et al. Treatment of amatoxin poisoning: 20-year retrospective analysis. $J$ Toxicol Clin Toxicol 2002;40:715-57.

6. Diaz JH. Syndromic diagnosis and management of confirmed mushroom poisonings. Crit Care Med 2005;33:427-36.

7. Larrey D, Pageaux GP. Hepatotoxity of herbal remedies and mushrooms. Semin Liver Dis 1995;15:183-8.

8. Ward J, Kapadia K, Brush E, et al. Amatoxin poisoning: case reports and review of current therapies. J Emerg Med 2013; 44:116-21.

9. Gores KM, Hamieh TS, Schmidt GA. Survival following investigational treatment of amanita mushroom poisoning: Thistle or shamrock? Chest 2014; 146:e126-9.

10. Tong TC, Hernandez M, Richardson WH III, et al. Comparative treatment of alpha-amanitin poisoning with $\mathrm{N}$-acetylcysteine, benzylpenicillin, cimetidine, thioctic acid, and silybin in a murine model. Ann Emerg Med 2007;50:282-8.

Affiliations: Department of Medicine (Stein, Wu), University of Toronto; Division of Clinical Pharmacology and Toxicology (Wu, Scott), Sunnybrook Health Sciences Centre; Dalla Lana School of Public Health (Scott), University of Toronto; Division of General Internal Medicine (Weinerman), Sunnybrook Health Sciences Centre, Toronto, Ont.

Contributors: All of the authors made substantial contributions to this work. Adina Weinerman contributed to design and conception of this report. Corey Stein and Peter Wu wrote the first draft of the manuscript. All of the authors contributed to the critical review of the literature, edited and revised the manuscript, and approved the final version to be published. James Scott identified the mushroom and provided photographs of the specimens.

Acknowledgement: The authors thank Margaret Thompson for her involvement as the poison control physician and for reviewing the case report. 